\title{
Is there a relationship between hand grip strength and knee osteoarthritis in terms of radiological and functional findings in female patients?
}

\author{
Fazıl Kulaklı@, İlker Fatih Sarı@ \\ Department of Physical Medicine and Rehabilitation, Giresun University Faculty of Medicine, Giresun, Turkey
}

\begin{abstract}
Objectives: The aim of this study was to assess the presence of the relationship between hand grip strength (HGS) and radiological and functional signs of knee osteoarthritis (KOA).

Patients and methods: Between March 2019 and January 2020, a total of 64 female patients (mean age: $63.4 \pm 8.8$ years; range, 50 to 80 years) with bilateral chronic knee pain who were diagnosed with KOA using radiological and clinical findings according to the 2019 American College of Rheumatology guidelines were included in the study. Patient demographics, body mass index (BMI) values, and hand dominance were recorded. The Western Ontario and McMaster Universities Arthritis Index (WOMAC) and Lower Extremity Functional Scale (LEFS) were used for the assessment of KOA functionality. The Kellgren-Lawrence (KL) grading system was used for the radiological assessment of KOA. Electronic dynamometer was used for HGS measurement and values were normalized according to BMI results.

Results: In the assessment of relationship between HGS and functionality of KOA, a negative, moderate correlation was found between the WOMAC and HGS-Dominant (D) $(r=0.312, p<0.05)$ and HGS-Non-Dominant (ND) $(r=0.391, p<0.01)$. In addition, a positive, moderate correlation was found between the LEFS and HGS-D $(r=0.344, p<0.01)$ and HGS-ND $(r=0.371, p<0.01)$. There was a weak, negative correlation between the HGS-ND, KL-D $(r=0.256, p<0.05)$ and KL-ND $(r=0.283, p<0.05)$, while no significant correlation was found between the HGS-D and KL.

Conclusion: Our study results show that HGS-ND is associated with KOA radiologically and functionally. The HGS should be added in the WOMAC and LEFS scales in the functional assessment of KOA and new scales including HGS assessment should be developed.

Keywords: Hand grip strength, muscle, osteoarthritis.
\end{abstract}

Knee osteoarthritis (KOA) is a chronic degenerative disease of the knee joint that progresses with age and severely affects knee joint functionality, as well as daily life activities. The symptomatic KOA prevalence is $14.8 \%$ in adults over 50 years of age, and it is $22.5 \%$ in females and $8 \%$ in males in Turkey. ${ }^{1}$ Although there are a number of risk factors associated with KOA, weakness in lower extremity muscles, particularly in quadriceps muscle, was shown to play a major role in the development of symptomatic KOA. ${ }^{2,3}$ However, there are contradicting results regarding the relationship of Quadrupedal Movement (QM) strength and radiological $\mathrm{KOA}$ in the literature. ${ }^{4,5}$

The hand grip strength (HGS) is a preferred method which is positively correlated with QM

Received: November 04, 2020 Accepted: December 16, 2020 Published online: February 08, 2021

Correspondence: Fazıl Kulaklı, MD. Giresun Üniversitesi Tıp Fakültesi Fiziksel Tıp ve Rehabilitasyon Anabilim Dalı, 28100 Giresun, Türkiye. Tel: +90 505 - 3121016 e-mail: drfzl46@gmail.com

\section{Citation:}

Kulaklı F, Sarı IF. Is there a relationship between hand grip strength and knee osteoarthritis in terms of radiological and functional findings in female patients? Arch Rheumatol 2021;36(3):389-397. 
strength and used in the assessment of muscle strength required for sarcopenia diagnosis. ${ }^{6,7}$ Patients with weak QM and/or sarcopenic obesity are reported to be under a higher risk of KOA development. 8,9 Therefore, HGS assessment can be beneficial in evaluating the functional and radiological results of KOA. There is only one study examining the HGS relation of radiological $\mathrm{KOA}$; however, the radiological evaluation method used in this study is not applicable in routine practice. ${ }^{10}$ No study is available evaluating the relationship of HGS with functionality of KOA in the literature.

In the present study, we hypothesized that HGS measurement could be related to KOA as radiologically and functionally and, thus, it could be implemented in functional and radiological assessment of KOA due to its simplicity, cost-effectiveness, and ease of application. In this study, we, therefore, aimed to assess the presence of the relationship between HGS and radiological and functional signs of KOA using objective measurement tools.

\section{PATIENTS AND METHODS}

The prospective study was conducted at Giresun University Faculty of Medicine, Department of Physical Medicine and Rehabilitation between March 2019 and January 2020. A total of 83 female patients were included in the study who presented to clinic for bilateral chronic knee pain (>3 months) and diagnosed with KOA using radiological and clinical findings according to the 2019 American College of Rheumatology (ACR) guidelines. ${ }^{11}$ Patients over 50 years old were included in the study to obtain an age and sex standard. No upper limit was set for age. Exclusion criteria were as follows: weakness or paresthesia in upper-lower extremity $(n=3)$; pain or sensory symptoms on lower-upper extremity (except for knee) $(n=2)$; muscle atrophy in upper-lower extremity muscles (except for bilateral QM atrophy due to KOA) $(n=1)$; a pathology on deep tendon reflexes or sensory examination $(n=2)$; trauma history within the past three months which might affect HGS and knee extension strength (KES) $(n=1)$; uncontrolled diabetes mellitus with polyneuropathy $(n=1)$; cervical-lumbar disk hernia $(n=1)$; cervical-lumbar spinal stenosis $(\mathrm{n}=2)$; nerve compression syndromes of upper-lower extremities $(n=3)$; upper-lower extremity surgery history $(n=3)$; active infection and malignancies; patients under medication which might affect muscle strength or pain level (muscle relaxants, analgesics, gabapentinoids, etc.); and having injection to knee or hand region within the past three months or receiving physical therapy. A total of 19 patients who met criteria were excluded from the study and, finally, 64 female patients (mean age: $63.4 \pm 8.8$ years; range, 50 to 80 years) completed the study. A written informed consent was obtained from each patient. The study protocol was approved by the Giresun University Faculty of Medicine Ethics Committee (No. KAEK-110). The study was conducted in accordance with the principles of the Declaration of Helsinki.

All assessments were done in face-to-face interviews. Patients' demographic characteristics, systemic diseases, body mass index (BMI), current medication use, tobacco and alcohol use, educational status, and dominant hand side were recorded. The Western Ontario and McMaster Universities Arthritis Index (WOMAC) and Lower Extremity Functional Scale (LEFS) were used for functional assessment, whereas the Kellgren-Lawrence (KL) grading system was used for radiological assessment of KOA. The HGS and KES were measured using electronic dynamometers.

\section{Instruments}

The WOMAC is a valid and reliable method used in the assessment of knee and hip osteoarthritis (OA) patients. The Outcome Measures in Rheumatology Clinical Trials (OMERACT) also recommends using this scale for OA studies. The scale consists of three parts which assess pain, stiffness, and physical function including 24 questions. Higher WOMAC scores indicate a greater level of pain and stiffness with decreased physical functioning. ${ }^{12}$ In Turkey, Tüzün et al. $^{13}$ performed the validity and reliability studies of the Turkish translation of WOMAC.

The Lower Extremity Functional Scale is a self-reported scale which can be filled out by 
the patients to assess the functional status of lower extremities in the presence of an issue with musculoskeletal system. ${ }^{14}$ The validity and reliability studies of the LEFS in the Turkish population were conducted by Citaker et al. ${ }^{15}$ Higher LEFS scores indicate positive physical functioning.

Radiological KOA was assessed using KL grading system ${ }^{16}$ with knee X-rays within the past month as follows:

\section{Stage 0: Normal}

Stage 1: Suspicious osteophytes, normal joint space

Stage 2: Significant osteophyte, suspicious narrowing of joint space

Stage 3: Moderate grade osteophytes, moderate narrowing of joint space and slight sclerosis

Stage 4: Big osteophytes, advanced joint space narrowing, significant subchondral bone sclerosis and cysts. The KL was staged by an experienced researcher on both dominant (-D) and non-dominant (-ND) knees, who was blind to the study population.

The HGS was measured using an electronic hand dynamometer (Camry, SCACAMEH10117; Zhongshan Camry Electronic Co, Ltd., Zhongshan, Guangdong, China) bilaterally. The patients were positioned in a neutral shoulder position, with the elbow in 90 degrees flexion and in semi-pronation. The measurements were taken three times, with $30 \mathrm{sec}$ resting period after each measurement, ${ }^{17}$ and maximum values were recorded in Newtons.

The KES was measured using a Lafayette Model 01165 (Lafayette Company Inc., Lafayette IN, USA) hand-held dynamometer. For the QM measurement point, the midline point of $5 \mathrm{~cm}$ above the lateral malleolus, which was shown as valid and reliable in previous studies, was defined and bilateral measurement was performed. ${ }^{18}$ Similar to HGS measurements, the measurements were taken three times with $30-\mathrm{sec}$ intervals between each measurement. A force was applied from measurement point at least for $3 \mathrm{sec}$, and measured maximum values were recorded in Newtons. Both HGS and KES measurements were taken by a single researcher to obtain a standardization of the measurements.
Since BMI could affect the measured muscle strength values, they were normalized according to BMI values. In this context, the muscle strength was calculated in Newtons, as the measurements were taken using a dynamometer and the measured value was divided $\mathrm{BMI}^{2 / 3}$ to obtain the normalized muscle strength. All muscle strength measurements found within the study and the tables were expressed in normalized muscle strength values (Normalized muscle strength $=$ Muscle strength measured in Newton/BMI $\left.{ }^{2 / 3}\right){ }^{19}$

\section{Statistical analysis}

Statistical analysis was performed using the IBM SPSS version 21.0 software (IBM Corp., Armonk, NY, USA). Descriptive statistics were expressed in mean \pm standard deviation (SD), median (min-max) or number and percentage. The Shapiro-Wilk test and normal quantile plots were utilized to assess the normal distribution of the variables. The Pearson correlation was used to identify the relationship between dominant and non-dominant HGS and KES among each other, as well as functional assessment. The relation of KL-D and KL-ND with the muscle strength and functional assessment scores was examined using the Spearman rho correlation. A coefficient value of $r \leq 0.30$ was deemed as a weak, $r>0.30$ to $\leq 0.70$ was deemed as a moderate, and $r>0.70$ was deemed as a strong relationship. ${ }^{20}$ For the relationship between HGS-D, HGS-ND and other variables, a linear regression analysis was performed. A $p$ value of $<0.05$ was considered statistically significant.

\section{RESULTS}

Patients' clinical and demographic characteristics are given in Table 1.

Patients' D and ND values of the HGS and KES and their correlations with functional assessment scores are shown in Table 2 . According to the correlation analysis between KES and HGS, there was a moderate, positive correlation between the HGS-D and KES-D $(\mathrm{r}=0.539, \mathrm{p}<0.01)$ and KES-ND $(\mathrm{r}=0.572$, $\mathrm{p}<0.01)$. Also, there was a moderate, positive correlation with the HGS-ND and KES-D $(r=0.700, \quad p<0.01)$ and KES-ND $(r=0.680$, 
Table 1. Demographic and clinical properties of patients $(n=64)$

\begin{tabular}{|c|c|c|c|}
\hline & $\mathrm{n}$ & $\%$ & Mean \pm SD \\
\hline Age (year) & & & $63.4 \pm 8.8$ \\
\hline Body mass index $\left(\mathrm{kg} / \mathrm{m}^{2}\right)$ & & & $32.2 \pm 4.9$ \\
\hline $\begin{array}{l}\text { Education } \\
\text { Primary education } \\
\text { Secondary education } \\
\text { High school-college }\end{array}$ & $\begin{array}{l}19 \\
35 \\
10\end{array}$ & $\begin{array}{l}29.7 \\
54.7 \\
15.6\end{array}$ & \\
\hline $\begin{array}{l}\text { Smoker } \\
\text { Yes } \\
\text { No }\end{array}$ & $\begin{array}{c}8 \\
56\end{array}$ & $\begin{array}{l}12.5 \\
87.5\end{array}$ & \\
\hline $\begin{array}{l}\text { Dominant/side } \\
\text { Right } \\
\text { Left }\end{array}$ & $\begin{array}{r}59 \\
5\end{array}$ & $\begin{array}{c}92.2 \\
7.8\end{array}$ & \\
\hline $\begin{array}{l}\text { Normalized muscle strengt } \\
\text { HGS-D } \\
\text { HGS-ND } \\
\text { KES-D } \\
\text { KES-ND }\end{array}$ & & & $\begin{array}{l}20.6 \pm 6.3 \\
19.8 \pm 6.0 \\
17.7 \pm 6.0 \\
15.0 \pm 5.8\end{array}$ \\
\hline $\begin{array}{l}\text { KL-D } \\
1 \\
2 \\
3 \\
4\end{array}$ & $\begin{array}{c}10 \\
26 \\
19 \\
9\end{array}$ & $\begin{array}{l}15.6 \\
40.6 \\
29.7 \\
14.1\end{array}$ & \\
\hline $\begin{array}{c}\text { KL-ND } \\
1 \\
2 \\
3 \\
4\end{array}$ & $\begin{array}{r}11 \\
35 \\
13 \\
5\end{array}$ & $\begin{array}{c}17.2 \\
54.7 \\
20.3 \\
7.8\end{array}$ & \\
\hline WOMAC & & & $49.6 \pm 18.7$ \\
\hline LEFS & & & $37.7 \pm 14.1$ \\
\hline $\begin{array}{l}\text { SD: Standard deviation; HG } \\
\text { ND: Non-dominant side; KE } \\
\text { Lawrence; WOMAC: Western } \\
\text { Index; LEFS: Lower Extremit } \\
\text { muscle strength in Newtons by }\end{array}$ & $\begin{array}{l}\text { ip str } \\
\text { tensio } \\
\text { d McN } \\
\text { l Scal } \\
\text { / ( } \mathrm{kg}\end{array}$ & $\begin{array}{l}\text { gth; D: } \\
\text { strength } \\
\text { ter Uni } \\
{ }^{*} \text { Calcu } \\
\text { 2) }\end{array}$ & $\begin{array}{l}\text { Ominant side; } \\
\text { KL: Kellgren- } \\
\text { sities Arthritis } \\
\text { ed by dividing }\end{array}$ \\
\hline
\end{tabular}

$p<0.01)$ with a higher coefficient. Table 2 also shows the assessment of the relationship between muscle strength and functional assessment scales. Accordingly, the HGSD, HGS-ND, KES-D, and KES-ND showed a moderate, negative correlation with the WOMAC scores and a moderate, positive significant correlation with the LEFS scores.

The correlation between the KL and muscle strength with functional assessment scales is shown in Table 3. There was a mild, negative, but significant correlation between the KL-D and HGS-ND ( $\mathrm{r}=-0.256, \mathrm{p}<0.01)$ and KES-D $(\mathrm{r}=-0.270, \quad \mathrm{p}<0.01)$. However, no significant correlations were found between the $\mathrm{KL}$ and HGS-D, KES-ND, WOMAC, and LEFS scores. There was a mild, negative correlation between the KL-ND and HGS-ND $(r=-0.283, p<0.01)$, although no significant correlation was found with the other parameters.

In addition to the correlation analysis, a linear regression analysis was performed to examine a possible relationship between HGS-D, HGS-ND and KES-D, KES-ND, WOMAC, and LEFS (Table 4). The scatter plot of HGS-D, HGS-ND on KES-D, KES-ND, WOMAC and LEFS with regression line and \%95 confidence interval (CI) are also shown in Figures 1 and 2. There was a statistically significant relationship between the HGS-D

Table 2. Pearson correlation analysis of normalized muscle strength values and functional assessment scales

\begin{tabular}{|c|c|c|c|c|c|c|}
\hline Muscle strength ${ }^{* * * *}$ & HGS-D & HGS-ND & KES-D & KES-ND & WOMAC & LEFS \\
\hline HGS-D & & $0.861^{* *}$ & $0.539^{* * *}$ & $0.572^{* * *}$ & $-0.312^{*}$ & $0.344^{* * *}$ \\
\hline HGS-ND & & & $0.700^{* * *}$ & $0.680^{* *}$ & $-0.391^{* * *}$ & $0.371^{* * *}$ \\
\hline KES-D & & & & $0.805^{* * *}$ & $-0.405^{* *}$ & $0.336^{* * *}$ \\
\hline KES-ND & & & & & $-0.453^{* *}$ & $0.380^{* * *}$ \\
\hline
\end{tabular}

HGS: Hand grip strength; D: Dominant side; ND: Non-dominant side; KES: Knee extension strength; WOMAC: Western Ontario and McMaster Universities Arthritis Index; LEFS: Lower Extremity Functional Scale; * Significance $\mathrm{p}<0.05$; ${ }^{*}$ Significance $\mathrm{p}<0.01$; *** Calculated by dividing muscle strength in Newtons by $\mathrm{BMI}^{2 / 3}(\mathrm{~N}) /\left(\mathrm{kg} / \mathrm{m}^{2}\right)^{2 / 3}$.

Table 3. Spearman correlation analysis of K-L grading system with normalized muscle strength values** and functional assessment scales

\begin{tabular}{lcccccc}
\hline KL scale & HGS-D & HGS-ND & KES-D & KES-ND & WOMAC & LEFS \\
KL-D & -0.210 & $-0.256^{*}$ & $-0.270^{*}$ & -0.121 & -0.213 \\
KL-ND & -0.167 & $-0.283^{*}$ & -0.174 & -0.124 & 0.238 & -0.201 \\
\hline
\end{tabular}

HGS: Hand grip strength; D: Dominant side; ND: Non-dominant side; KES: Knee extension strength; WOMAC: Western Ontario and McMaster Universities Arthritis Index; LEFS: Lower Extremity Functional Scale; * Significance $p<0.05 ;{ }^{* *}$ Calculated by dividing muscle strength in Newtons by $\mathrm{BMI}^{2 / 3}(\mathrm{~N}) /\left(\mathrm{kg} / \mathrm{m}^{2}\right)^{2 / 3}$. 
Table 4. Linear regression analysis between dominant and non-dominant hand grip strength

\begin{tabular}{|c|c|c|c|c|c|c|c|c|}
\hline \multirow[b]{2}{*}{ Variables } & \multicolumn{4}{|c|}{ HGS-D } & \multicolumn{4}{|c|}{ HGS-ND } \\
\hline & B (\%95 CI) & SE & $\mathrm{R}^{2}$ & $p$ & B (\%95 CI) & SE & $\mathrm{R}^{2}$ & $p$ \\
\hline KES-D & $0.570(0.343-0.796)$ & 0.113 & 0.290 & $<0.001$ & $0.706(0.523-0.889)$ & 0.092 & 0.489 & $<0.001$ \\
\hline KES-ND & $0.622(0.395-0.849)$ & 0.113 & 0.316 & $<0.001$ & $0.706(0.513-0.900)$ & 0.097 & 0.463 & $<0.001$ \\
\hline WOMAC & $-0.105(-0.187--0.024)$ & 0.041 & 0.097 & 0.012 & $-0.126(-0.201--0.051)$ & 0.038 & 0.153 & 0.001 \\
\hline LEFS & $0.154(0.047-0.261)$ & 0.053 & 0.119 & 0.005 & $0.159(0.058-0.259)$ & 0.050 & 0.138 & 0.003 \\
\hline
\end{tabular}

HGS: Hand grip strength; D: Dominant side; ND: Non-dominant side; B: Coefficient of linear regression; CI: Confidence interval; SE: Standard error; $\mathrm{R}^{2}$ : Coefficient of determination; KES: Knee extension strength; WOMAC: Western Ontario and McMaster Universities Arthritis Index; LEFS: Lower Extremity Functional Scale.
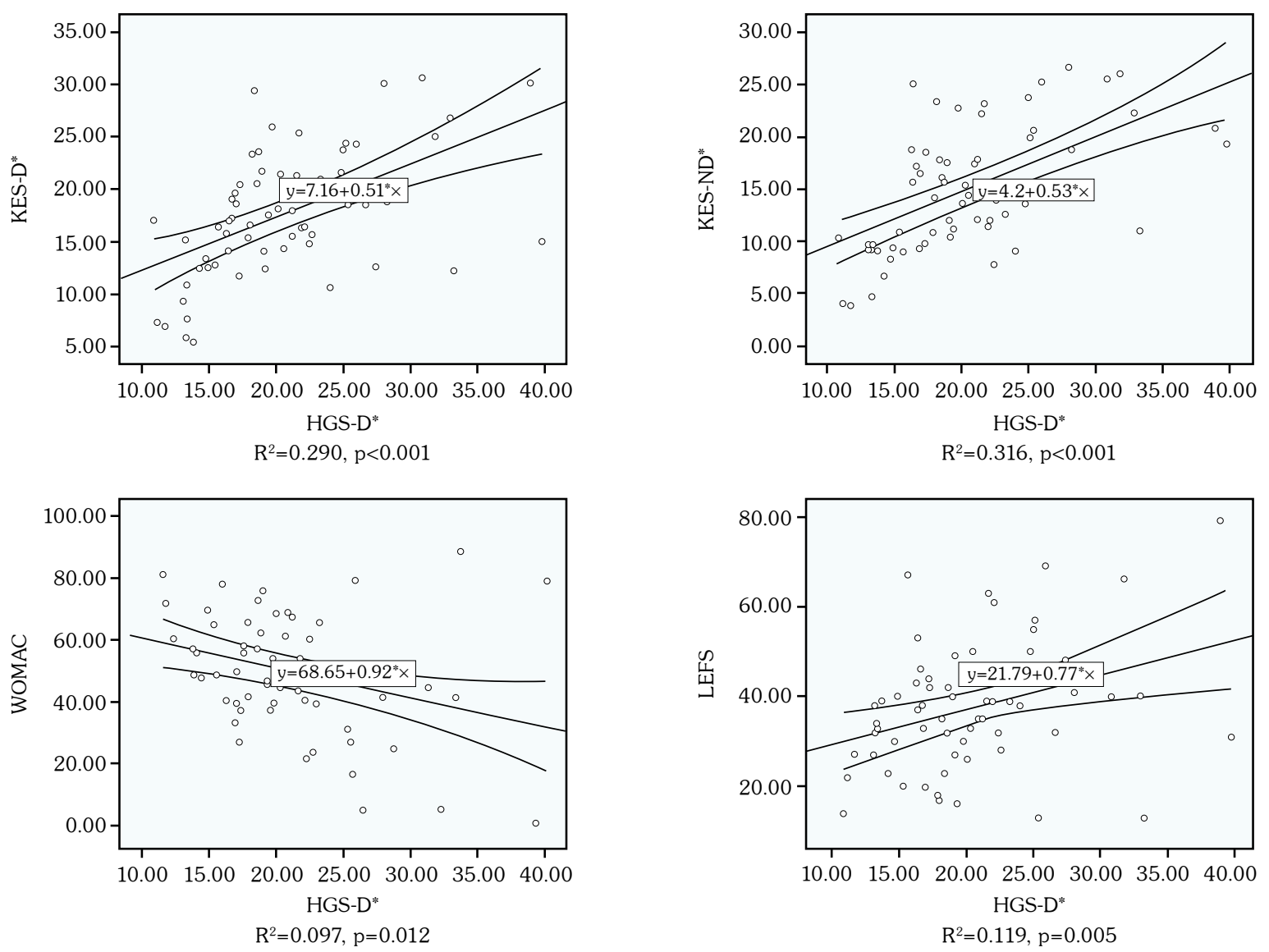

Figure 1. Relationship between HGS-D and variables tested (KES-D, KES-ND, WOMAC and LEFS) with scatter plot.

HGS: Hand grip strength; D: Dominant side; ND: Non-dominant side; KES: Knee extension strength; WOMAC: Western Ontario and McMaster Universities Artritis Index; LEFS: Lower Extremity Functional Scale; ${ }^{2}$ : Coefficient of determination; * Calculated by dividing muscle strength in Newtons by $\mathrm{BMI}^{2 / 3}(\mathrm{~N}) /\left(\mathrm{kg} / \mathrm{m}^{2}\right)^{2 / 3}$.

and KES-D $\left(\mathrm{R}^{2}=0.290, \mathrm{p}<0.001\right)$, KES-ND $\left(\mathrm{R}^{2}=0.316, \quad \mathrm{p}<0.001\right)$, WOMAC $\left(\mathrm{R}^{2}=0.097\right.$, $\mathrm{p}=0.012)$, LEFS $\left(\mathrm{R}^{2}=0.119, \mathrm{p}=0.005\right)$. Also there was a statistically significant relationship between the HGS-ND and KES-D $\left(\mathrm{R}^{2}=0.489\right.$, $\mathrm{p}<0.001), \quad$ KES-ND $\left(\mathrm{R}^{2}=0.463, \quad \mathrm{p}<0.001\right)$, WOMAC $\quad\left(\mathrm{R}^{2}=0.153, \quad \mathrm{p}=0.001\right), \quad$ LEFS $\left(\mathrm{R}^{2}=0.138, \mathrm{p}=0.003\right)$. 

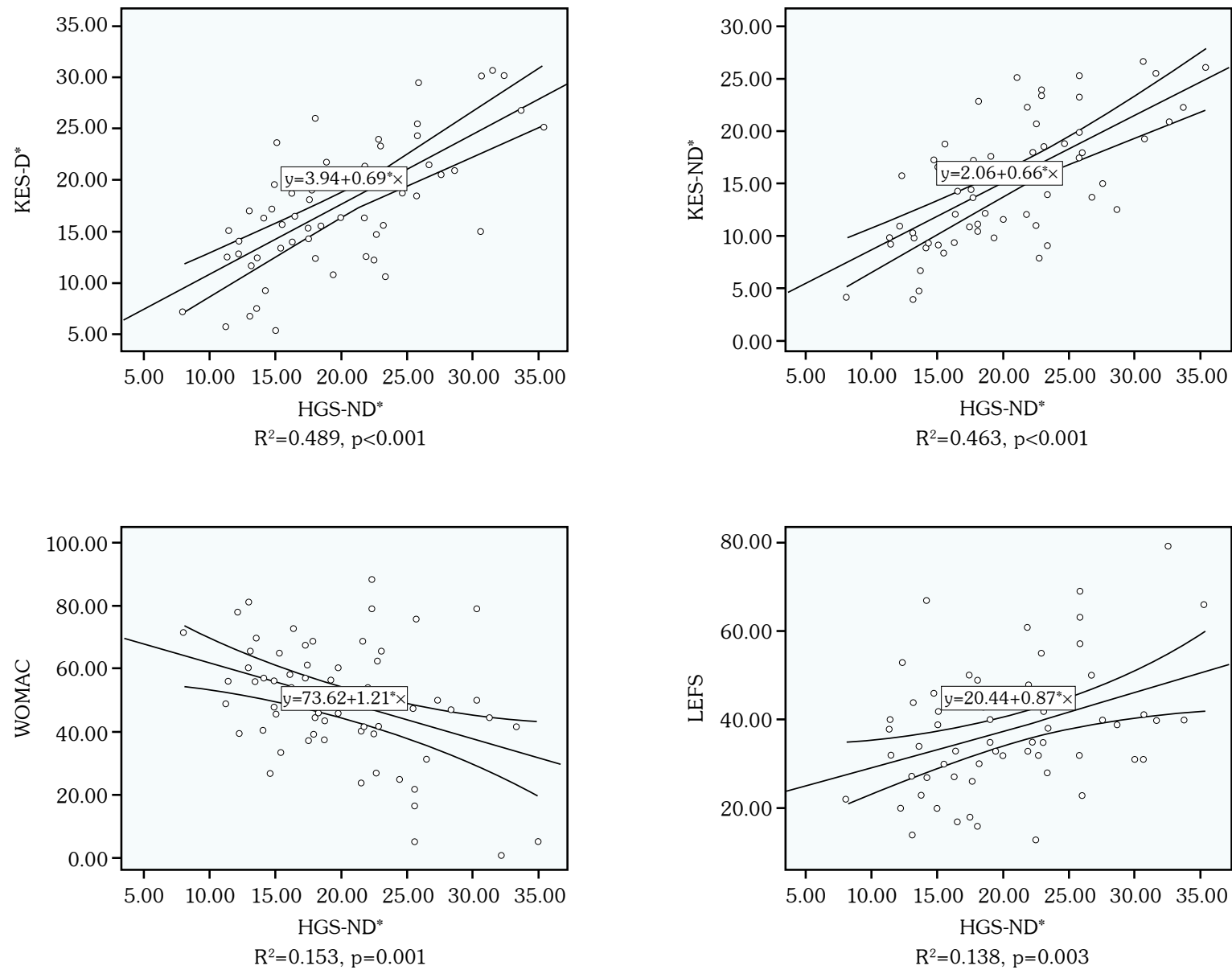

Figure 2. Relationship between HGS-ND and variables tested (KES-D, KES-ND, WOMAC and LEFS) with scatter plot.

HGS: Hand grip strength; D: Dominant side; ND: Non-dominant side; KES: Knee extension strength; WOMAC: Western Ontario and McMaster Universities Artritis Index; LEFS: Lower Extremity Functional Scale; $\mathrm{R}^{2}$ : Coefficient of determination; ${ }^{*}$ Calculated by dividing muscle strength in Newtons by $\mathrm{BMI}^{2 / 3}(\mathrm{~N}) /\left(\mathrm{kg} / \mathrm{m}^{2}\right)^{2 / 3}$.

\section{DISCUSSION}

Although the main question which comes to mind in this study is that how a method assessing the hand can have a relationship with KOA, it should be kept in mind that HGS has a positive correlation with QM strength and HGS is the most recommended assessment tool for muscle strength in the algorithmic approach for sarcopenia diagnosis. ${ }^{6}$ Moreover, weak QM and sarcopenic obesity are known risk factors for KOA development, as previously reported in the literature. ${ }^{9}$ In the light of these data, we hypothesized that HGS could be related to KOA radiologically and functionally, leading us to conduct this study. As a result of this study, the HGS-ND showed a significant correlation with both radiological and functional parameters, supporting our hypothesis. Therefore, application of HGS, in particular HGS-ND, which is a cost-effective, easily accessible, and easy-to-use method, seems to be overlooked in the clinical and radiological severity assessment of $\mathrm{KOA}$, despite being an important parameter. We, therefore, suggest that scales used to measure functional status in KOA should also include HGS values.

Another point that makes this study unique is that it is the first to analyze the HGS relation with KOA in terms of functional parameters. Additionally, this study is the first to assess the HGS relation with radiological KOA, as evidenced by KL grading. Finally, another important feature of this study which sets it apart from other studies 
is that the measured muscle strength values were normalized using normalization formulas found in the literature to eliminate BMI effect. ${ }^{19}$

In the literature, there are a number of studies examining the relation of HGS with hand OA. Those studies reported a negative, but significant correlation between HGS and radiological and symptomatic hand OA. ${ }^{21,22}$ However, there are controversial studies showing the opposite and reporting that increased HGS also increased the hand OA development risk. ${ }^{23}$ Although there are many studies examining the relationship between HGS and hand OA, there is only one study investigating the relationship between HGS and KOA. ${ }^{10}$ In this study, the relationship between HGS and radiological hand OA and KOA was examined and weak HGS was related to more severe radiological hand $\mathrm{OA}$ and $\mathrm{KOA}$ results. ${ }^{10}$ Again, KL was not used for radiological KOA assessment and the authors used a radiological scale they developed for the study, instead. Although we used different radiological assessment methods in our study, Wen et al. ${ }^{10}$ showed similar results: i.e., a negative, but significant correlation between the HGS and radiological KOA. However, our study found a significant relationship only between the HGS-ND with KL-D and KL-ND and none with HGS-D. Different from Wen et al.'s study, ${ }^{10}$ patients with conditions which might affect HGS, and also those having findings during examinations that were thought to affect HGS were excluded, to effectively eliminate the risk of contradicting results with the measurement of HGS values. Another different point of this study is that the muscle strength values were normalized using BMI and the effect of BMI over muscle strength was eliminated which allowed us to examine the pure muscle strength. Therefore, it was able to obtain more reliable results by assessing the muscle strength in a more clarified setting, free from secondary causes.

The HGS and knee extension torque were shown to have a strong relationship between each other, as reported in the literature, and HGS has a strong correlation with the lower extremity strength. ${ }^{7}$ Also, in our study, a moderate, positive, and significant correlation was found between the HGS-D and HGS-ND with KES-D and KES-ND. The HGS-ND and KES-D and KES-ND had also a moderate, positive, and significant correlation with higher correlation coefficients. The weakness of
QM was found to be associated with an increased radiological severity in KOA in previous studies; ${ }^{24}$ however, whether it has a significant effect is still unclear. On the other hand, the QM strength is still considered as an important parameter in KOA management. ${ }^{4}$ Consistent with these contradicting results, our study also showed a weak, negative, but significant correlation between the KES-D and KL-D, yet KES-ND and KL showed no relation at all. Of note, we consider that $\mathrm{QM}$ strength is a crucial parameter in radiological and functional management of KOA. Based on these data, we suggest that normalized HGS-ND measurements should be used more often in both KES and radiological KOA assessments. This is due to the fact that dominant hand is usually stronger, as it is used more in the daily tasks, thereby, leading to incorrect results in the measurements and, thus, HGS-ND is more suitable for the assessment in radiological KOA and KES.

There are studies showing increased functionality and decreased pain in KOA patients with stronger $\mathrm{QM},{ }^{8}$ which is consistent with the results of this study. However, there are no previous studies examining the relationship between HGS and KOA functionality. In our study, we observed a significant correlation between the functionality of KOA with both HGS-D and HGS-ND. To sum up, HGS measurement, which is an easy and applicable method, can be considered a valuable tool in the functional assessment of KOA. On the other hand, the WOMAC and LEFS scales, that are used in the functional assessment of KOA, which were also used in this study, are not helpful for HGS assessments. Thus, we believe that questions which can evaluate the HGS should be included in these scales or new scales including HGS assessment should be developed.

Although sarcopenia presence is not shown as a clear risk factor for KOA development, sarcopenic obesity is a defined risk factor for KOA in the literature. ${ }^{9}$ Yet, it is evident that sarcopenia can cause weakness in QM, which is also proven that decreased QM strength is a risk factor for KOA development. ${ }^{4}$ The HGS measurement is the most important method recommended in the algorithmic approach for sarcopenia diagnosis. ${ }^{6,7}$ Therefore, the importance of HGS measurement in sarcopenia assessment becomes evident. Although HGS, on its own, is not enough to make a definitive sarcopenia 
diagnosis, it is still important as a measurement method to distinguish sarcopenia. Since KOA and sarcopenia are usually seen in similar age groups, for the clinical assessment for sarcopenia and KOA, particularly in individuals over 50 years of age, HGS measurement should be used more frequently.

Although not being the main objective of this study, no significant relationship was found between radiological KOA and functionality of $\mathrm{KOA}$, as previously reported in the literature. ${ }^{25}$ This finding indicates that evaluation of radiological results alone is not enough for a clear assessment of functional status of the patients.

In this study, only female patients were included to provide a better standardization, which can be considered the main limitation.

In conclusion, our study results show that HGS-ND is associated with KOA radiologically and functionally. The HGS should be added in the WOMAC and LEFS scales in the functional assessment of $\mathrm{KOA}$ and new scales including HGS assessment should be developed.

\section{Declaration of conflicting interests}

The authors declared no conflicts of interest with respect to the authorship and/or publication of this article.

\section{Funding}

The authors received no financial support for the research and/or authorship of this article.

\section{REFERENCES}

1. Kaçar C, Gilgil E, Urhan S, Arikan V, Dündar U, Oksüz MC, et al. The prevalence of symptomatic knee and distal interphalangeal joint osteoarthritis in the urban population of Antalya, Turkey. Rheumatol Int 2005;25:201-4.

2. Omori G, Koga Y, Tanaka M, Nawata A, Watanabe $\mathrm{H}$, Narumi K, et al. Quadriceps muscle strength and its relationship to radiographic knee osteoarthritis in Japanese elderly. J Orthop Sci 2013;18:536-42.

3. Segal NA, Glass NA, Torner J, Yang M, Felson DT, Sharma $\mathrm{L}$, et al. Quadriceps weakness predicts risk for knee joint space narrowing in women in the MOST cohort. Osteoarthritis Cartilage 2010;18:769-75.

4. Segal NA, Glass NA. Is quadriceps muscle weakness a risk factor for incident or progressive knee osteoarthritis? Phys Sportsmed 2011;39:44-50.
5. Takagi S, Omori G, Koga H, Endo K, Koga Y, Nawata A, et al. Quadriceps muscle weakness is related to increased risk of radiographic knee OA but not its progression in both women and men: the Matsudai Knee Osteoarthritis Survey. Knee Surg Sports Traumatol Arthrosc 2018;26:2607-14.

6. Chen LK, Liu LK, Woo J, Assantachai P, Auyeung TW, Bahyah KS, et al. Sarcopenia in Asia: consensus report of the Asian Working Group for Sarcopenia. J Am Med Dir Assoc 2014;15:95-101.

7. Cruz-Jentoft AJ, Baeyens JP, Bauer JM, Boirie Y, Cederholm T, Landi F, et al. Sarcopenia: European consensus on definition and diagnosis: Report of the European Working Group on Sarcopenia in Older People. Age Ageing 2010;39:412-23.

8. Bennell KL, Wrigley TV, Hunt MA, Lim BW, Hinman $\mathrm{RS}$. Update on the role of muscle in the genesis and management of knee osteoarthritis. Rheum Dis Clin North Am 2013;39:145-76.

9. Misra D, Fielding RA, Felson DT, Niu J, Brown $\mathrm{C}$, Nevitt $\mathrm{M}$, et al. Risk of knee osteoarthritis with obesity, sarcopenic obesity, and sarcopenia. Arthritis Rheumatol 2019;71:232-7.

10. Wen L, Shin MH, Kang JH, Yim YR, Kim JE, Lee $\mathrm{JW}$, et al. Association between grip strength and hand and knee radiographic osteoarthritis in Korean adults: Data from the Dong-gu study. PLoS One 2017;12:e0185343.

11. Altman R, Asch E, Bloch D, Bole G, Borenstein $\mathrm{D}$, Brandt $\mathrm{K}$, et al. Development of criteria for the classification and reporting of osteoarthritis. Classification of osteoarthritis of the knee. Diagnostic and Therapeutic Criteria Committee of the American Rheumatism Association. Arthritis Rheum 1986;29:1039-49.

12. Angst F, Aeschlimann A, Steiner W, Stucki G. Responsiveness of the WOMAC osteoarthritis index as compared with the SF-36 in patients with osteoarthritis of the legs undergoing a comprehensive rehabilitation intervention. Ann Rheum Dis 2001;60:834-40.

13. Tüzün EH, Eker L, Aytar A, Daşkapan A, Bayramoğlu M. Acceptability, reliability, validity and responsiveness of the Turkish version of WOMAC osteoarthritis index. Osteoarthritis Cartilage 2005;13:28-33.

14. Yeung TS, Wessel J, Stratford P, Macdermid J. Reliability, validity, and responsiveness of the lower extremity functional scale for inpatients of an orthopaedic rehabilitation ward. J Orthop Sports Phys Ther 2009;39:468-77.

15. Citaker S, Kafa N, Hazar Kanik Z, Ugurlu M, Kafa B, Tuna Z. Translation, cross-cultural adaptation and validation of the Turkish version of the Lower Extremity Functional Scale on patients with knee injuries. Arch Orthop Trauma Surg 2016;136:389-95.

16. Kellgren JH, Lawrence JS. Radiological assessment of osteo-arthrosis. Ann Rheum Dis 1957;16:494-502. 
17. Minetto MA, Caresio C, Menapace T, Hajdarevic A, Marchini A, Molinari F, et al. Ultrasound-based detection of low muscle mass for diagnosis of sarcopenia in older adults. PM R 2016;8:453-62.

18. Gellhorn AC, Stumph JM, Zikry HE, Creelman CA, Welbel R. Ultrasound measures of muscle thickness may be superior to strength testing in adults with knee osteoarthritis: a cross-sectional study. BMC Musculoskelet Disord 2018;19:350.

19. Jaric S. Role of body size in the relation between muscle strength and movement performance. Exerc Sport Sci Rev 2003;31:8-12.

20. Büyüköztürk Ş. Sosyal bilimler için veri analizi el kitabı. 13. Bask1. Ankara: Pegem Akademi; 2011. s. 92.

21. Haugen IK, Slatkowsky-Christensen B, Bøyesen $\mathrm{P}$, van der Heijde D, Kvien TK. Cross-sectional and longitudinal associations between radiographic features and measures of pain and physical function in hand osteoarthritis. Osteoarthritis Cartilage 2013;21:1191-8.

22. Ding H, Solovieva S, Vehmas T, Takala EP, LeinoArjas P. Hand osteoarthritis and pinch grip strength among middle-aged female dentists and teachers. Scand J Rheumatol 2010;39:84-7.

23. Chaisson CE, Zhang Y, Sharma L, Kannel W, Felson DT. Grip strength and the risk of developing radiographic hand osteoarthritis: results from the Framingham Study. Arthritis Rheum 1999;42:33-8.

24. Omori G, Koga Y, Tanaka M, Nawata A, Watanabe $\mathrm{H}$, Narumi K, et al. Quadriceps muscle strength and its relationship to radiographic knee osteoarthritis in Japanese elderly. J Orthop Sci 2013;18:536-42.

25. Creamer P, Lethbridge-Cejku M, Hochberg MC. Factors associated with functional impairment in symptomatic knee osteoarthritis. Rheumatology (Oxford) 2000;39:490-6. 